\title{
Third-line antiretroviral therapy in Africa: effectiveness in a Southern African retrospective cohort study
}

\author{
Graeme Meintjes ${ }^{1,2,3^{*}}$, Liezl Dunn ${ }^{4}$, Marla Coetsee ${ }^{4}$, Michael Hislop ${ }^{4}$, Rory Leisegang ${ }^{5}$, Leon Regensberg ${ }^{4}$ \\ and Gary Maartens ${ }^{5}$
}

\begin{abstract}
Background: An increasing number of patients in Africa are experiencing virologic failure on second-line antiretroviral therapy (ART) and those who develop resistance to protease inhibitors (PI) will require third-line ART, but no data on the outcomes of third-line are available from the region. We assessed the virologic outcomes and survival of patients started on salvage ART in a Southern African private sector disease management programme.

Methods: Retrospective observational cohort study with linkage to the national death register. Adults ( $\geq 18$ years) who started salvage ART between July 2007 and December 2011 were included. Salvage ART was defined by inclusion of darunavir or tipranavir in an ART regimen after having failed another PI. For Kaplan-Meier (KM) analysis, patients were followed up until event, or censored at death (only for virologic outcomes), leaving the programme, or April 2014.

Results: 152 patients were included. Subtype was known for 113 patients: 111 (98\%) were infected with subtype C. All 152 had a genotype resistance test demonstrating major PI resistance mutations. Salvage drugs included were: darunavir/ritonavir $(n=149)$, tipranavir/ritonavir $(n=3)$, raltegravir $(n=58)$, and etravirine $(n=8)$. Median follow-up was 2.5 years $(\mathrm{IQR}=1.5-3.3) .82 .9 \%$ achieved a viral load $\leq 400 \mathrm{copies} / \mathrm{ml}$ and $71.1 \% \leq 50 \mathrm{copies} / \mathrm{ml}$. By the end of the study 17 (11.2\%) of the patients had died. The KM estimate of cumulative survival was $87.2 \%$ at 2000 days.

Conclusions: Virologic suppression was comparable to that demonstrated in clinical trials and observational studies of salvage ART drugs conducted in other regions. Few deaths occurred during short term follow-up. Third-line regimens for patients with multidrug resistant subtype C HIV in Africa are virologically and clinically effective.
\end{abstract}

Keywords: Virologic failure, Human immunodeficiency virus, HIV, Antiretroviral therapy, Salvage, Third-line

\section{Background}

The emergence of resistance to antiretroviral drugs is an inevitable consequence of expanding access to antiretroviral therapy (ART) and longer durations of exposure. Currently less than $15 \%$ of patients in ART programmes in Africa are on second-line ritonavirboosted protease inhibitor (PI) regimens $[1,2]$, but this

\footnotetext{
*Correspondence: graemein@mweb.co.za

${ }^{1}$ Clinical Infectious Diseases Research Initiative (CIDRI), Institute of Infectious Disease and Molecular Medicine (IDM), Faculty of Health Sciences, University of Cape Town, Anzio Road, Observatory, Cape Town 7925, South Africa

Full list of author information is available at the end of the article
}

proportion will increase significantly over time. Recent studies have shown that most patients failing secondline ART in resource-limited settings do not have major PI resistance-associated mutations, implying that failure is due to poor adherence rather than the development of resistance [3, 4]. However, with more patients failing on second-line ART regimens for longer durations this will likely change [5]. The threat of "untreatable" multidrug resistant HIV after second-line failure in Africa was recently highlighted [6].

The 2013 World Health Organization (WHO) ART guidelines recommended that national programmes should develop policies and conduct pilot studies for 
third-line ART for patients with multi-class resistance after second-line failure [7]. Newer ART salvage regimens, which include drugs such as darunavir, raltegravir and etravirine, have been associated with reasonable virologic suppression rates in clinical trials [8-10], but clinical outcome data are limited. Variation in drug resistance patterns and susceptibility have been reported in relation to HIV subtype differences [11-13], but no data exists on effectiveness of salvage regimens in Africa where the majority of patients are infected with subtype C.

We evaluated virologic and clinical outcomes on salvage ART in the Southern African private health care sector amongst patients who had previously experienced virologic failure on a PI-containing regimen and in whom a genotype antiretroviral resistance test (GART) prior to starting salvage ART demonstrated at least one major PI mutation. Our broader aim was to inform advocacy and planning for third-line ART in programmes in sub-Saharan Africa.

\section{Results}

On 31 December 2011 there were a total of 96,527 adult patients ( $\geq 18$ years) receiving ART in the Aid for AIDS (AfA) programme, 152 of whom fulfilled our inclusion criteria for salvage therapy. The characteristics of our cohort are shown in Table 1. One-hundred and forty patients reported being exposed to an NNRTI or had this recorded on the AfA database. Eight patients gave no history of NNRTI exposure and there was no record of NNRTI exposure on the AfA database (two of whom had NNRTI resistance mutations). In four patients NNRTI exposure history was not available because they joined the AfA programme only after they had been treated with ART outside of the programme and history of their original regimens was not available on the database; in two of these patients NNRTI resistance mutations were detected. All patients had failed a PI regimen $(97 \%$ failed a ritonavir-boosted PI regimen). A total of 185 GARTs were performed prior to salvage therapy: 123 patients had one, 25 had 2 and 4 had 3 GARTs. The subtype was recorded for 113 patients: 111/113 (98\%) were subtype C.

The following salvage drugs were prescribed: darunavir/ritonavir $(\mathrm{n}=149)$, tipranavir/ritonavir $(\mathrm{n}=3)$, raltegravir $(\mathrm{n}=58)$ and etravirine $(\mathrm{n}=8)$. NRTI drugs were included in 149 salvage regimens: tenofovir (TDF)/ emtricitabine (FTC) in 105, TDF/FTC/zidovudine (AZT) in 22, AZT/lamivudine (3TC) in 9, and other NRTI combinations in 13. In 2 patients although salvage therapy was authorised it could not be verified from documentation available that they had been dispensed salvage therapy. These 2 patients were, however, included in the analyses as we could not determine that they definitely had not been dispensed salvage therapy.

The median duration of follow-up on salvage therapy was 2.5 years $(\mathrm{IQR}=1.5-3.3)$. By date of administrative censor (30 April 2014), 17 of 152 patients (11.2\%) had died. 145/152 (95.4\%) patients had at least 1 viral load done on salvage therapy: $126 / 145$ achieved a VL $\leq 400$ copies/ml (86.9 \%) and 108/145 achieved a VL $\leq 50$ copies $/ \mathrm{ml}$ (74.5\%). Of all 152 patients, $82.9 \%$ were therefore documented to have suppressed VL $\leq 400$ copies $/ \mathrm{ml}$ and $71.1 \% \mathrm{VL} \leq 50$ copies/ml. Kaplan-Meier (KM) estimates of cumulative proportions of all 152 patients suppressing to a $\mathrm{VL} \leq 400$ copies $/ \mathrm{ml}$ at 1000 days was $92.2 \%$ and $\mathrm{VL} \leq 50 \mathrm{copies} / \mathrm{ml}$ was 83.4 \% (Fig. 1a, b). Factors associated with VL suppression $\leq 50$ copies/ml are shown in Table 2. In multivariate analysis, we found women were more likely to suppress $(\mathrm{aOR}=2.45,95 \% \mathrm{CI}=1.07-$ 5.63), whereas patients with higher VL and higher levels of darunavir resistance were less likely to suppress $\left(\mathrm{aOR}=0.38,95 \% \mathrm{CI}=0.21-0.70\right.$ per $\log _{10}$ increase in $\mathrm{VL}$, and $\mathrm{aOR}=0.59,95 \% \mathrm{CI}=0.39-0.90$ per 10 point increase on Stanford database, respectively). There was a non-significant trend towards the inclusion of raltegravir in the salvage regimen being associated with a higher odds of suppression ( $\mathrm{aOR}=2.38,95 \% \mathrm{CI}=0.95-5.95$ ).

The cumulative incidence of virologic rebound after suppression was 19 and $13.9 \%$ of those suppressing to VL $\leq 400$ and $\leq 50$ copies $/ \mathrm{ml}$ respectively. The median CD 4 count increased from 153 to 448 cells $/ \mathrm{mm}^{3}$ by 3 years on salvage ART (Fig. 2). The KM estimate of cumulative survival was $87.2 \%$ at 2000 days (Fig. 1c). One patient stopped ritonavir of their own accord due to gastro-intestinal side effects. There were no other interruptions or substitutions for intolerance or toxicity.

\section{Discussion}

A high proportion of these Southern African private sector patients on ART salvage therapy for a median of 2.5 years achieved virologic suppression. Factors independently associated with virologic suppression were female sex, lower darunavir resistance score, lower VL at start of salvage ART, and use of raltegravir (although the latter just failed to achieve statistical significance). The KM estimate of survival at 2000 days was $87 \%$. These results are striking given that this cohort of patients had previously failed multiple lines of therapy.

The proportion of patients achieving virologic suppression in our study was near to that achieved in the ANRS 139 TRIO trial conducted in France in which patients with multiclass resistant HIV were treated with darunavir, raltegravir, etravirine and either NRTIs or enfuvirtide. In that single-arm trial, $88 \%$ of participants achieved virologic suppression (VL $<50$ copies/ml) at 96 weeks 
Table 1 Demographic and clinical characteristics and genotype antiretroviral resistance test profile of the 152 patients when starting salvage ART

\begin{tabular}{|c|c|}
\hline Characteristic & Number (\%) or median (IQR) \\
\hline \multicolumn{2}{|l|}{ Demographic } \\
\hline Female gender & $75(49 \%)$ \\
\hline Age (years) & $44(39.8-48)$ \\
\hline \multicolumn{2}{|l|}{ Clinical } \\
\hline CD4 count when initially started ART (cells/mm³) $(n=109)$ & $102(31-186)$ \\
\hline HIV VL when initially started ART (copies/ml) $(n=100)$ & $176,893(73,425-500,000)$ \\
\hline CD4 count when started salvage ART (cells $\left./ \mathrm{mm}^{3}\right)(n=152)$ & $153(41-329)$ \\
\hline HIV VL when started salvage ART (copies/ml) $(n=152)$ & $82,831(20,060-233,778)$ \\
\hline Number of ART regimens prior to salvage ART & $3(3-4)$ \\
\hline Known to have originally initiated ART with dual NRTI regimen & $53(34.9 \%)$ \\
\hline Duration of ART exposure prior to salvage ART (years) $(n=122)^{a}$ & $8.9(6.9-10.4)$ \\
\hline Duration of PI exposure prior to salvage ART (years) $(n=148)^{b}$ & $5.1(3.4-7.2)$ \\
\hline \multicolumn{2}{|l|}{ Genotype antiretroviral resistance test $(\text { GART) })^{c}$} \\
\hline \multicolumn{2}{|l|}{ Thymidine analogue mutations ${ }^{\mathrm{d}, \mathrm{e}}$} \\
\hline 0 & $45(30 \%)$ \\
\hline $1-2$ & $35(23 \%)$ \\
\hline$\geq 3$ & $72(47 \%)$ \\
\hline \multicolumn{2}{|l|}{ Major protease inhibitor mutations ${ }^{f}$} \\
\hline $1-2$ & $38(25 \%)$ \\
\hline$\geq 3$ & $114(75 \%)$ \\
\hline \multicolumn{2}{|l|}{ Stanford resistance scores } \\
\hline Lopinavir & $70(40-90)$ \\
\hline Darunavir & $10(0-20)$ \\
\hline Etravirine $^{g}$ & $5(0-15)$ \\
\hline Lopinavir score > 29 (intermediate/high level resistance) & $136(89.5 \%)$ \\
\hline Lopinavir score > 59 (high level resistance) & $88(57.9 \%)$ \\
\hline Darunavir score > 29 (intermediate/high level resistance) & $14(9.2 \%)$ \\
\hline Darunavir score > 59 (high level resistance) & $0(0.0 \%)$ \\
\hline Etravirine score $>29$ (intermediate/high level resistance) & $28(18.4 \%)$ \\
\hline Etravirine score > 59 (high level resistance) & $7(4.6 \%)$ \\
\hline
\end{tabular}

147 were treated in South Africa, 4 in Swaziland and 1 in Namibia

$A R T$ antiretroviral therapy, IQR interquartile range; NRTI nucleoside reverse transcriptase inhibitor, PI protease inhibitor, VL viral load

a In 30 insufficient data on ART history was available to calculate ART duration

b In 4 insufficient data on PI history was available to calculate ART duration

c If a patient had more than one GART, then resistance mutations detected in any GART were included in these analyses

d Thymidine analogue mutations were defined as: M41L, D67N, K70R, L210W, T215Y/F and K219Q/E

e Other major nucleoside reverse transcriptase inhibitor mutations present were: M184V $(n=84), \operatorname{K} 65 R(n=7), L 74 V(n=11), Q 151 M(n=4)$ and T69 insertion $(n=1)$

f Major protease inhibitor mutations were defined as: D30N, V32I, M46I/L, 147V/A, G48V/M/A/S/T/Q/L, I50L/V, I54V/T/A/S/L/M, L76V, V82A/C/T/S/F/L/M, I84V/A/C, N88S/T/G and L90M

${ }^{g}$ Few patients were taking non-nucleoside reverse transcriptase inhibitor drugs at the time the pre-salvage GART was performed

[14]. While a clinical trial may represent a select patient group, several observational studies of patients with multiclass resistant HIV treated with darunavir-based regimens have also reported virologic suppression (VL $<50$ copies/ml by week 48) being achieved in over $50 \%$ of patients. This includes studies conducted in other developing countries: Brazil (83\% suppression [15] and $73 \%$ suppression [16]) and Mexico (69 \% suppression [17]).
The inclusion of raltegravir in such darunavir-based regimens has been associated with improved virologic outcomes $[16,18]$.

The need for evidence regarding the implementation of third-line ART in resource-limited settings has been recognized by the WHO [7]. Our data demonstrated the effectiveness of third-line ART in a programme where the predominant subtype was $C$, in contrast to clinical 


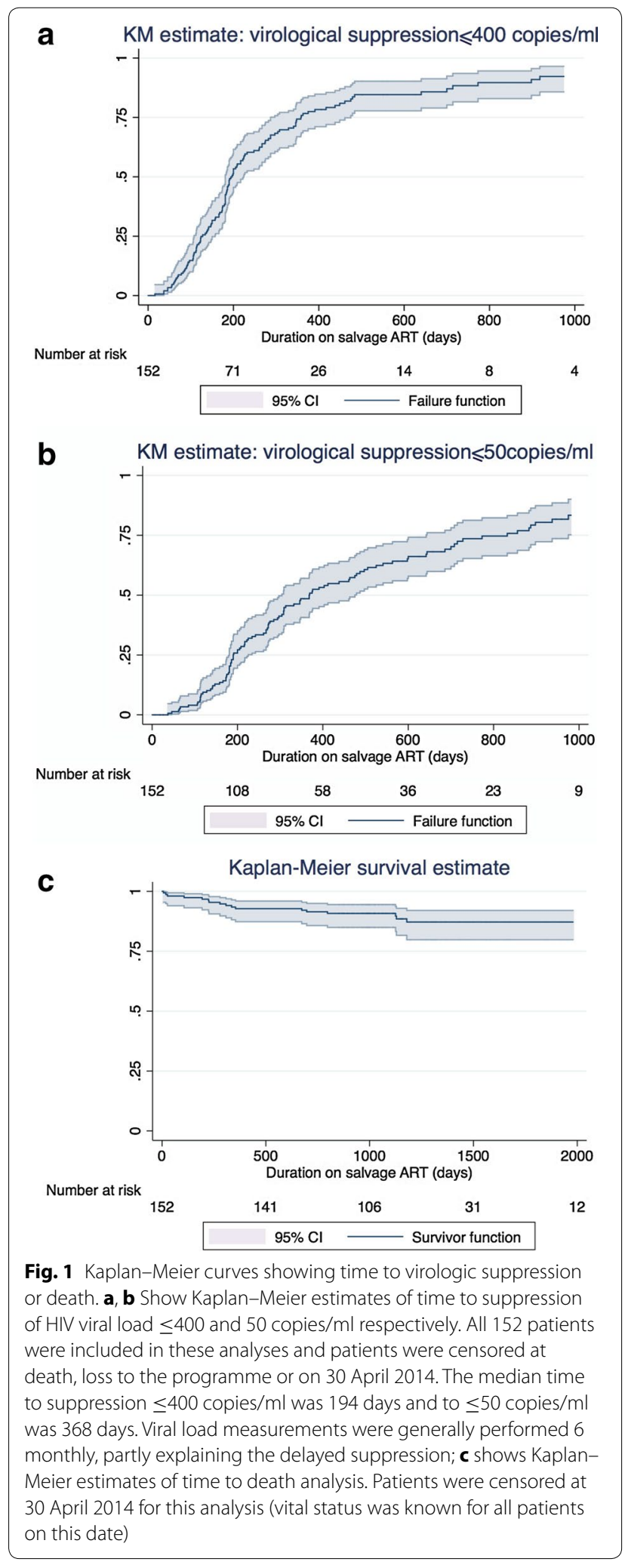

trials of salvage drugs done in settings where non- $C$ subtypes predominate. Our findings are in line with those of prior studies, which suggested that raltegravir and darunavir regimens had equivalent efficacy in patients with $B$ and non-B subtype infections and that resistance to these drugs was not more prevalent with non-B subtypes [19-23]. The fact that the majority of patients achieved virologic suppression on regimens including darunavir/ ritonavir, raltegravir and NRTIs suggested this could be a standardized third-line regimen, with individual adjustments based on GART.

Important limitations of our study were the retrospective observational study design, which could have resulted in selection and information bias, and potential limited generalizability to the public sector. While there are important socioeconomic differences between private and public sector patients in the region, which could affect outcomes, we felt that our results are applicable to public sector programmes as similar treatment guidelines were followed and 5 year outcomes were previously reported to be similar between AfA and a large South African public sector programme [24]. Of note is that the South African public sector ART programme has recently approved third-line therapy using similar selection criteria to AfA's. A key strength of our study is that we reported on clinical outcomes.

\section{Conclusions}

AfA has been providing ART, GART and new salvage drug options for longer than public sector programmes, thus providing us with the first opportunity to assess outcomes on salvage drugs in an African setting. These data provide the first evidence of the potential effectiveness of third-line regimens based largely upon a backbone of darunavir/ritonavir and raltegravir together with NRTI drugs, in a predominantly subtype $C$ African epidemic. Availability of GART and darunavir/ritonavir and raltegravir (or dolutegravir) needs to be planned for the future as more patients fail second-line PI regimens for longer durations in public sector programmes on the continent. Our results provide evidence that third-line regimens for patients failing second-line ART with multidrug resistant HIV in Africa are likely to be effective.

\section{Methods}

\section{Design}

We conducted a retrospective observational cohort study of adults ( $\geq 18$ years) enrolled with the Aid for AIDS (AfA) 
Table 2 Multivariate logistic regression analysis of factors associated with HIV viral load suppression $\leq 50$ copies/ml on salvage ART

\begin{tabular}{|c|c|c|c|c|}
\hline Variable & $\begin{array}{l}\text { Unadjusted odds ratio } \\
(95 \% \mathrm{Cl})\end{array}$ & p value & $\begin{array}{l}\text { Adjusted odds ratio } \\
(95 \% \mathrm{Cl})\end{array}$ & p value \\
\hline Age (per year increase) & $1.00(0.95-1.05)$ & 0.96 & $1.00(0.94-1.06)$ & 0.98 \\
\hline Female gender & $2.42(1.16-5.01)$ & 0.02 & $2.45(1.07-5.63)$ & 0.04 \\
\hline HIV viral load at start of salvage ART (per log10 unit increase) & $0.30(0.17-0.53)$ & $<0.001$ & $0.38(0.21-0.70)$ & 0.002 \\
\hline $\begin{array}{l}\text { Darunavir resistance score (per } 10 \text { point increase on Stanford } \\
\text { database) }\end{array}$ & $0.54(0.38-0.76)$ & $<0.001$ & $0.59(0.39-0.90)$ & 0.01 \\
\hline Raltegravir in salvage regimen & $1.11(0.54-2.30)$ & 0.77 & $2.38(0.95-5.95)$ & 0.06 \\
\hline
\end{tabular}

a All 152 patients are included in this analysis. Those who did not have a viral load performed or who died before having a suppressed viral load recorded were regarded as having failed to suppress

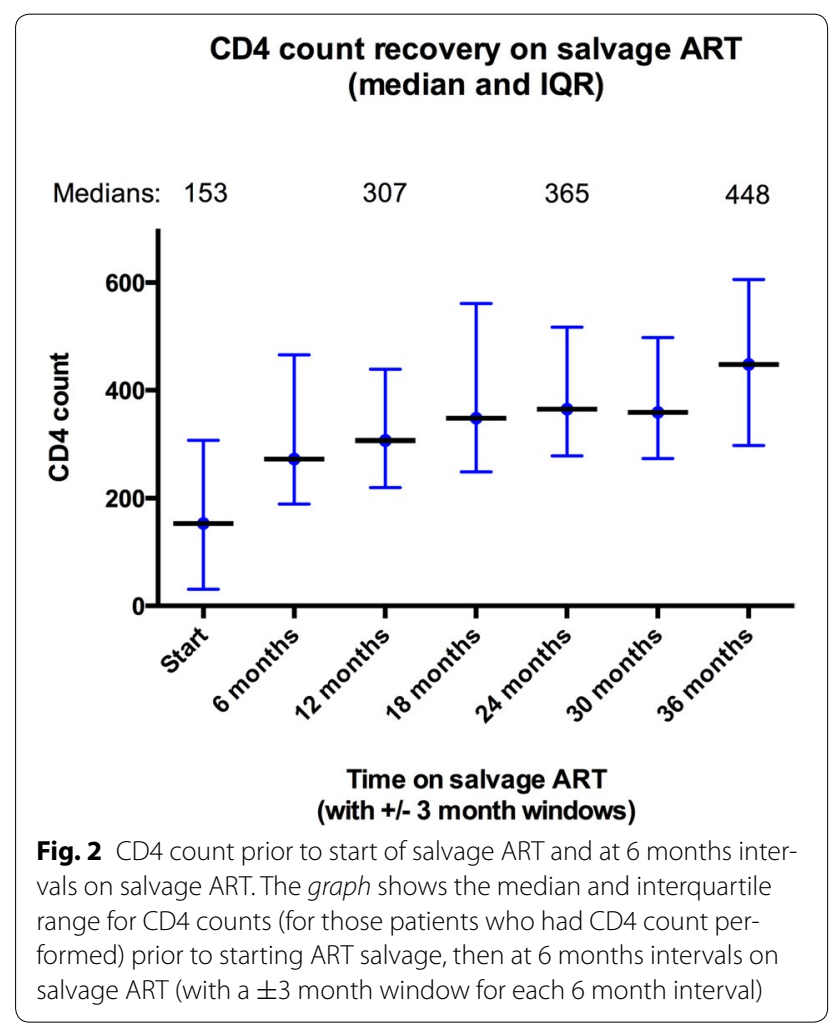

disease management programme, who started salvage ART between 1 July 2007 and 31 December 2011. Salvage ART was defined by inclusion of darunavir or tipranavir in an ART regimen after having experienced virologic failure on another PI. No patients had prior exposure to an integrase inhibitor. All patients had a GART performed prior to starting a salvage ART regimen that was used to inform choice of drugs in this regimen. These GARTs were performed at quality-assured private sector laboratories and results were sent to the patient's doctor and to AfA. Certain laboratories reported the HIV subtype on the laboratory results sheet routinely, but for some patients $(n=39)$ this information was not supplied. Salvage drugs that became available during the study period were initially darunavir and later raltegravir and etravirine-these were prescribed to patients commencing salvage regimens after they became available if indicated, but were not added as single drugs for a patient already on salvage ART. Access to tipranavir was very limited.

\section{Setting}

AfA is a private sector HIV disease management programme that provides HIV care for patients on medical insurance and corporate treatment programmes [25, 26]. HIV care includes clinical interventions coupled with clinical and patient support and on-going adherence management. Although it operates in the private sector, the AfA guidelines are similar to the WHO guidelines with a standardized approach to first-line (NNRTI-based) and second-line (protease inhibitor-based) ART regimens. Demographic and clinical data are captured in the AfA database. Salvage ART was authorized by a clinical committee after reviewing treatment history and GART. All patients starting salvage ART were contacted before being switched to assess commitment and educate them about the regimen, and thereafter monthly for adherence support until viral load was suppressed.

Important differences between AfA and the public sector ART programme in South Africa are: (1) the AfA programme started in 1998 whereas the public sector programme started in 2004; (2) in AfA some patients received initial ART with dual NRTIs for affordability reasons in the late 1990s; (3) certain drugs were available in the private sector before the public sector; (4) GART has been available for patients failing ART in AfA since 2001; and (5) darunavir was readily available to patients on the AfA programme from 2007, and raltegravir from 2009. These two drugs became available in the public sector programme in 2013.

Commencement of salvage ART was checked against the pharmacy claims history on the AfA database. Vital status was available for all patients at the date of data 
censor: (1) for South Africans through linkage to the South African national death register and (2) for patients outside South Africa from the clinical records. The Stanford HIV Drug Resistance Database (version 6.2.0) was used to calculate mutation scores.

\section{Statistical analyses}

For Kaplan-Meier (KM) analyses of time to virologic suppression or death, patients were followed up until the date of the event or censored at death (only for virologic outcomes), leaving the programme, or date of administrative censor (30 April 2014). Multivariate logistic regression analysis was performed using STATA 13, StataCorp, TX, US, with all variables hypothesised to be associated with outcome included in the model. Virologic rebound was defined as $\geq 1$ viral load $(\mathrm{VL})>1000$ copies/ $\mathrm{ml}$ after initial suppression.

\section{Authors' contributions}

GM and GM designed the study; LD, MC, MH and RL extracted and collated data for analysis; GM, LD, MC and MH conducted data analysis; and interpretation of data was conducted by GM, LD, MH, LR and GM. GM drafted the manuscript which was then revised and finalized with contributions from all authors. All authors read and approved the final manuscript.

\section{Author details}

${ }^{1}$ Clinical Infectious Diseases Research Initiative (CIDRI), Institute of Infectious Disease and Molecular Medicine (IDM), Faculty of Health Sciences, University of Cape Town, Anzio Road, Observatory, Cape Town 7925, South Africa. 2 Division of Infectious Diseases and HIV Medicine, Department of Medicine, University of Cape Town, Cape Town, South Africa. ${ }^{3}$ Department of Medicine, Imperial College London, London, UK. ${ }^{4}$ Aid for AIDS Management, Afrocentric Health Pty Ltd, The Boulevard, Block G, Searle Street, Woodstock 7925, South Africa. ${ }^{5}$ Division of Clinical Pharmacology, Department of Medicine, University of Cape Town, Groote Schuur Hospital Old Main Building, Observatory, Cape Town 7925, South Africa.

\section{Acknowledgements}

Graeme Meintjes was supported by a Wellcome Trust fellowship [098316] and in part by the National Research Foundation (NRF) of South Africa [UID 85858]. The grant holder acknowledges that opinions, findings and conclusions or recommendations expressed in any publication generated by the NRF supported research are that of the author(s), and that the NRF accepts no liability whatsoever in this regard. The funders played no role in design, in the collection, analysis, and interpretation of data; in the writing of the manuscript; nor in the decision to submit the manuscript for publication.

\section{Competing interests}

The authors declare that they have no competing interests.

\section{Ethical approval}

This was obtained from the UCT Human Research Ethics Committee (REF $557 / 2012)$

\section{Preliminary data from this study was previously presented as a poster abstract}

Dunn L, Coetsee M, Regensberg L, Hislop M, Maartens G, Meintjes G. Virological response to antiretroviral salvage therapy in the Southern African private sector. Southern African HIV Clinicians Society Conference, Cape Town, November 2012.

Received: 4 June 2015 Accepted: 16 November 2015

Published online: 01 December 2015

\section{References}

1. Boulle A, Van Cutsem G, Hilderbrand K, Cragg C, Abrahams M, Mathee S, Ford N, Knight L, Osler M, Myers J, et al. Seven-year experience of a primary care antiretroviral treatment programme in Khayelitsha. S Afr Aids. 2010;24(4):563-72.

2. Madec Y, Leroy S, Rey-Cuille MA, Huber F, Calmy A. Persistent difficulties in switching to second-line ART in sub-saharan Africa-a systematic review and meta-analysis. PLoS One. 2013;8(12):e82724.

3. Van Zyl GU, Liu TF, Claassen M, Engelbrecht S, de Oliveira T, Preiser W, Wood NT, Travers S, Shafer RW. Trends in genotypic HIV-1 antiretroviral resistance between 2006 and 2012 in South African patients receiving first- and second-line antiretroviral treatment regimens. PLoS One. 2013;8(6):e67188.

4. Wallis CL, Mellors JW, Venter WD, Sanne I, Stevens W. Protease inhibitor resistance is uncommon in HIV-1 subtype $C$ infected patients on failing second-line lopinavir/r-containing antiretroviral therapy in South Africa. AIDS Res Treat. 2011;2011:769627.

5. Rawizza HE, Chaplin B, Meloni ST, Darin KM, Olaitan O, Scarsi KK, Onwuamah CK, Audu RA, Chebu PR, Imade GE, et al. Accumulation of protease mutations among patients failing second-line antiretroviral therapy and response to salvage therapy in Nigeria. PLoS One. 2013;8(9):e73582.

6. Magambo B, Nazziwa J, Bbosa N, Gupta RK, Kaleebu P, Parry CM. The arrival of untreatable multidrug-resistant HIV-1 in sub-Saharan Africa. Aids. 2014:28(9):1373-4.

7. World Health Organization. Consolidated guidelines on the use of antiretroviral drugs for treating and preventing HIV infection: recommendations for a public health approach. Geneva: WHO; 2013

8. Arasteh K, Yeni P, Pozniak A, Grinsztejn B, Jayaweera D, Roberts A, Hoy J, De Meyer S, Vangeneugden T, Tomaka F. Efficacy and safety of darunavir/ ritonavir in treatment-experienced HIV type-1 patients in the POWER 1, 2 and 3 trials at week 96. Antivir Ther. 2009;14(6):859-64.

9. Steigbigel RT, Cooper DA, Teppler H, Eron JJ, Gatell JM, Kumar PN, Rockstroh JK, Schechter M, Katlama C, Markowitz M, et al. Long-term efficacy and safety of Raltegravir combined with optimized background therapy in treatment-experienced patients with drug-resistant HIV infection: week 96 results of the BENCHMRK 1 and 2 Phase III trials. Clin Infect Dis Off Publ Infect Dis Soc Am. 2010;50(4):605-12.

10. Yazdanpanah Y, Fagard C, Descamps D, Taburet AM, Colin C, Roquebert B, Katlama C, Pialoux G, Jacomet C, Piketty C, et al. High rate of virologic suppression with raltegravir plus etravirine and darunavir/ritonavir among treatment-experienced patients infected with multidrug-resistant HIV: results of the ANRS 139 TRIO trial. Clin Infect Dis Off Publ Infect Dis Soc Am. 2009;49(9):1441-9.

11. Sunpath H, Wu B, Gordon M, Hampton J, Johnson B, Moosa MY, Ordonez C, Kuritzkes DR, Marconi VC. High rate of K65R for antiretroviral therapynaive patients with subtype C HIV infection failing a tenofovir-containing first-line regimen. Aids. 2012;26(13):1679-84.

12. Wainberg MA, Zaharatos GJ, Brenner BG. Development of antiretroviral drug resistance. New Engl J Med. 2011;365(7):637-46.

13. Wainberg MA, Brenner BG. The Impact of HIV Genetic Polymorphisms and Subtype Differences on the Occurrence of Resistance to Antiretroviral Drugs. Molecular biology international. 2012;2012:256982.

14. Fagard C, Colin C, Charpentier C, Rami A, Jacomet C, Yeni P, Vittecoq D, Katlama C, Molina JM, Descamps D, et al. Long-term efficacy and safety of raltegravir, etravirine, and darunavir/ritonavir in treatment-experienced patients: week 96 results from the ANRS 139 TRIO trial. J Acquir Immune Defic Syndr. 2012;59(5):489-93.

15. Vidal JE, Song AT, Matos ML, Bartmann D, Anjos GD, Miranda EJ, Freitas AC, Dalben Mde F, Santana C, Segurado AC. High rate of virologic suppression with darunavir/ritonavir plus optimized background therapy among highly antiretroviral-experienced HIV-infected patients: results of a prospective cohort study in Sao Paulo, Brazil. Braz J Infect Dis Off Publ Braz Society Infect Dis. 2013;17(1):41-7.

16. Biscione FM, Westin MR, Ribeiro KM, Estevam DL, Cardoso SW, Tenore SB, Neto LF, Alencastro PR, Suffert TA, de Moraes MJ, et al. Virologic and immunologic effectiveness at 48 weeks of darunavir-ritonavir-based regimens in treatment-experienced persons living with HIV-1 infection in clinical practice: a multicenter Brazilian cohort. J Int Assoc Provid AIDS Care. 2014;13(1):63-8.

17. Mata-Marin JA, Huerta-Garcia G, Dominguez-Hermosillo JC, ChavezGarcia M, Banda-Lara MI, Nunez-Rodriguez N, Cruz-Herrera JE, 
Sandoval-Ramirez JL, Martinez-Abarca I, Villagomez-Ruiz AF, et al. Effectiveness and risk factors for virological outcome of darunavir-based therapy for treatment-experienced HIV-infected patients. AIDS Res Ther. 2015;12:31.

18. Delaugerre C, Buyck JF, Peytavin G, Viard JP, Chaix ML, Zucman D, Mortie E, Blanche S, Rouveix E, Force G, et al. Factors predictive of successful darunavir/ritonavir-based therapy in highly antiretroviral-experienced HIV-1-infected patients (the DARWEST study). J Clin Virol Off Publ Pan American Soc Clin Virol. 2010;47(3):248-52.

19. Anta L, Blanco JL, Llibre JM, Garcia F, Perez-Elias MJ, Aguilera A, Perez-Romero P, Caballero E, Vidal C, Canizares A, et al. Resistance to the most recent protease and non-nucleoside reverse transcriptase inhibitors across HIV-1 non-B subtypes. J Antimicrobial Chemother. 2013;68(9):1994-2002.

20. Bar-Magen T, Donahue DA, McDonough El, Kuhl BD, Faltenbacher VH, Xu H, Michaud V, Sloan RD, Wainberg MA. HIV-1 subtype B and C integrase enzymes exhibit differential patterns of resistance to integrase inhibitors in biochemical assays. Aids. 2010;24(14):2171-9.

21. Dierynck I, De Meyer S, Lathouwers E, Vanden Abeele C, Van De Casteele T, Spinosa-Guzman S, de Bethune MP, Picchio G. In vitro susceptibility and virological outcome to darunavir and lopinavir are independent of HIV type-1 subtype in treatment-naive patients. Antiviral Ther 2010;15(8):1161-9.

22. Poveda E, de Mendoza C, Parkin N, Choe S, Garcia-Gasco P, Corral A, Soriano $V$. Evidence for different susceptibility to tipranavir and darunavi in patients infected with distinct HIV-1 subtypes. Aids. 2008;22(5):611-6.

23. Rockstroh JK, Teppler H, Zhao J, Sklar P, Miller MD, Harvey CM, Strohmaier KM, Leavitt RY, Nguyen BY. Clinical efficacy of raltegravir against $B$ and non-B subtype HIV-1 in phase III clinical studies. Aids. 2011;25(11):1365-9.

24. Sarkin L, Leisegang R, Lesosky M, Hislop M, Walters L, Zutavern A, Maartens $\mathrm{G}$. Insurability and survival of lives living with HIV and other chronic diseases. Oral abstract 85-A. 30th International Conference on Actuaries, Washington, 2014

25. Leisegang R, Cleary S, Hislop M, Davidse A, Regensberg L, Little F, Maartens $G$. Early and late direct costs in a Southern African antiretroviral treatment programme: a retrospective cohort analysis. PLoS Med. 2009;6(12):e1000189.

26. Regensberg L, Hislop M. Aid for AIDS: a report back on more than 4 years of HIV/AIDS disease mangment in Southern Africa. South Afr J HIV Med. 2003;2:7-10.

\section{Submit your next manuscript to BioMed Central and we will help you at every step:}

- We accept pre-submission inquiries

- Our selector tool helps you to find the most relevant journal

- We provide round the clock customer support

- Convenient online submission

- Thorough peer review

- Inclusion in PubMed and all major indexing services

- Maximum visibility for your research

Submit your manuscript at www.biomedcentral.com/submit
() Biomed Central 